Proyecciones Journal of Mathematics Vol. 31, No 1, pp. 39-49, March 2012. Universidad Católica del Norte Antofagasta - Chile

\title{
An upper bound on the largest signless Laplacian of an odd unicyclic graph*
}

\author{
MACARENA COLLAO \\ UNIVERSIDAD CATÓLICA DEL NORTE, CHILE \\ PAMELA PIZARRO \\ UNIVERSIDAD CATÓLICA DEL NORTE, CHILE \\ and \\ OSCAR ROJO \\ UNIVERSIDAD CATÓLICA DEL NORTE, CHILE \\ Received: November 2011. Accepted : January 2012
}

\begin{abstract}
We derive an upper bound on the largest signless Laplacian eigenvalue of an odd unicyclic graph. The bound is given in terms of the largest vertex degree and the largest height of the trees obtained removing the edges of the unique cycle in the graph.
\end{abstract}

AMS classification : 05C50, 15A48.

Keywords : Laplacian matrix; signless Laplacian matrix; adjacency matrix; spectral radius; generalized Bethe tree.

${ }^{*}$ Work supported by Project Fondecyt 1100072, Chile. 


\section{Introduction}

Let $\mathcal{G}$ be a simple undirected connected graph of order $n$. Let $E(\mathcal{G})$ be the set of edges of $\mathcal{G}$. Let $A(\mathcal{G})$ be the adjacency matrix of $\mathcal{G}$ and let $D(\mathcal{G})$ be the diagonal matrix of vertex degrees. The Laplacian matrix and the signless Laplacian matrix of $\mathcal{G}$ are $L(\mathcal{G})=D(\mathcal{G})-A(\mathcal{G})$ and $Q(\mathcal{G})=$ $D(\mathcal{G})+A(\mathcal{G})$, respectively. The matrices $L(\mathcal{G})$ and $Q(\mathcal{G})$ are both positive semidefinite matrices.

Let $\mu_{1}(\mathcal{G})$ and $q_{1}(\mathcal{G})$ be the largest eigenvalues of $L(\mathcal{G})$ and $Q(\mathcal{G})$, respectively.

Let $\Delta$ be the maximum vertex degree of a graph $\mathcal{G}$. In [7], Hu proves that if $\mathcal{G}$ is a unicyclic graph then

$$
\mu_{1}(\mathcal{G}) \leq \Delta+2 \sqrt{\Delta-1}
$$

with equality if and only if $\mathcal{G}$ is the cycle $\mathcal{C}_{n}$ whenever $n$ is even.

We recall that the height of a rooted tree is the largest distance from the root to a pendant vertex.

The following invariant for a unicyclic graph $\mathcal{G}$ has been introduced in [10].

Definition 1. Let $\mathcal{G}$ be a unicyclic graph. Let $\mathcal{C}_{r}$ be the unique cycle in $\mathcal{G}$ and let $v_{1}, v_{2}, \ldots, v_{r}$ be the vertices of $\mathcal{C}_{r}$. The graph $\mathcal{G}-E\left(\mathcal{C}_{r}\right)$ is a forest of $r$ rooted trees $\mathcal{T}_{1}, \mathcal{T}_{2}, \ldots, \mathcal{T}_{r}$ with root vertices $v_{1}, v_{2}, \ldots ., v_{r}$, respectively. For $i=1,2, \ldots, r$, let $h\left(\mathcal{T}_{i}\right)$ be the height of the tree $\mathcal{T}_{i}$ with root $v_{i}$. Let

$$
k(\mathcal{G})=\max \left\{h\left(\mathcal{T}_{i}\right): 1 \leq i \leq r\right\}+1 .
$$

Example 1. For instance, if $\mathcal{G}$ is the graph

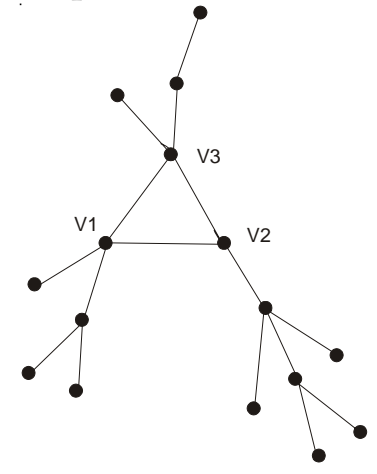

then $\Delta=4, h\left(\mathcal{T}_{1}\right)=2, h\left(\mathcal{T}_{2}\right)=3, h\left(\mathcal{T}_{3}\right)=2$ and $k(\mathcal{G})=\max \{2,3,2\}+$ $1=4$. 
In [10], the upper bound on $\mu_{1}(\mathcal{G})$ in (1.1) is improved by using the invariants $\Delta$ and $k(\mathcal{G})$. In fact, it is proved that if $\mathcal{G}$ is a unicyclic then

$$
\mu_{1}(\mathcal{G})<\Delta+2 \sqrt{\Delta-1} \cos \frac{\pi}{2 k(\mathcal{G})+1}
$$

for $\Delta \geq 3$.

The following lemma tell us that for any graph $\mathcal{G}$ the largest Laplacian eigenvalue does not exceed the largest signless Laplacian eigenvalue.

Lemma 1. $[2] \mu_{1}(\mathcal{G}) \leq q_{1}(\mathcal{G})$ with equality if and only if $\mathcal{G}$ is a bipartite graph.

In [5], a survey concerning upper bounds on $\mu_{1}(\mathcal{G})$ and $q_{1}(\mathcal{G})$ is given. Moreover, it is shown that many but not all upper bounds on $\mu_{1}(\mathcal{G})$ are also valid for $q_{1}(\mathcal{G})$. In this paper, we prove that if $\mathcal{G}$ is a unicyclic graph the upper bound on $\mu_{1}(\mathcal{G})$ in $(1.2)$ is also an upper bound on $q_{1}(\mathcal{G})$.

From now on, let $\mathcal{G}$ be a unicyclic graph. Let $\mathcal{C}_{r}$ be the unique cycle in $\mathcal{G}$. It is known that if $r$ is an even integer then $\mathcal{G}$ is a bipartite graph [3]. Moreover, if $\mathcal{G}$ is a bipartite graph then $Q(\mathcal{G})$ and $L(\mathcal{G})$ have the same eigenvalues [1]. Then, throughout this paper, we assume that $r$ is an odd integer, that is, that $\mathcal{G}$ is an odd unicyclic graph. If $\Delta=2$ then $\mathcal{G}=\mathcal{C}_{n}$ and thus $q_{1}(\mathcal{G})=q_{1}\left(\mathcal{C}_{n}\right)=4$. Then, we also assume that $\Delta \geq 3$.

Denote by $\sigma(M)$ and $\rho(M)$ the spectrum and the spectral radius of the matrix $M$, respectively.

The level of a vertex in a rooted tree is one more than its distance from the root vertex. A generalized Bethe tree is a rooted tree in which vertices at the same level have the same degree. Let $\mathcal{B}_{k}$ be a generalized Bethe tree of $k$ levels. For $j=1,2, \ldots, k$, let $d_{k-j+1}$ be the degree of the vertices of $\mathcal{B}_{k}$ at the level $j$ and let $n_{k-j+1}$ be the number of these vertices. Then, $n_{k}=1, d_{k}$ is the degree of the root, $n_{1}$ is the number of pendant vertices and $d_{1}=1$.

Let

$$
\Omega=\left\{j: 1 \leq j \leq k-1, n_{j}>n_{j+1}\right\}
$$

and, for $j=1,2, \ldots, k-1$, let

$$
T_{j}=\left[\begin{array}{cccc}
1 & \sqrt{d_{2}-1} & & \\
\sqrt{d_{2}-1} & d_{2} & \ddots & \\
& \ddots & \ddots & \sqrt{d_{j}-1} \\
& & \sqrt{d_{j}-1} & d_{j}
\end{array}\right] .
$$


In [11], one can find a complete characterization of the spectrum of the signless Laplacian matrix of a graph $\mathcal{H}\left\{\mathcal{B}_{k}\right\}$ obtained from a connected graph $\mathcal{H}$ of order $r$ and $r$ copies of $\mathcal{B}_{k}$ identifying the root of the $i-t h$ copy with the $i-t h$ vertex of $\mathcal{H}$. More precisely

Theorem 1. [11] If $\mathcal{H}$ is a connected graph of order $r$ then

$$
\sigma\left(Q\left(\mathcal{H}\left\{\mathcal{B}_{k}\right\}\right)\right)=\left(\cup_{j \in \Omega} \sigma\left(T_{j}\right)\right) \cup\left(\cup_{i=1}^{r} \sigma\left(S_{i}(\mathbf{d})\right)\right)
$$

where

$$
S_{i}(\mathbf{d})=\left[\begin{array}{ccccc}
1 & \sqrt{d_{2}-1} & & & \\
\sqrt{d_{2}-1} & d_{2} & \ddots & & \\
& \ddots & \ddots & \sqrt{d_{k-1}-1} & \\
& & \sqrt{d_{k-1}-1} & d_{k-1} & \sqrt{d_{k}} \\
& & & \sqrt{d_{k}} & d_{k}+q_{i}(\mathcal{H})
\end{array}\right]
$$

and

$$
q_{1}(\mathcal{H}) \geq q_{2}(\mathcal{H}) \geq \ldots \geq q_{r-1}(\mathcal{H}) \geq q_{r}(\mathcal{H})
$$

are the signless Laplacian eigenvalues of $\mathcal{H}$.

At this point, we recall that if $A$ is an irreducible nonnegative matrix then $A$ has an eigenvalue equal to its spectral radius $\rho(A)$ and $\rho(A)$ increases when any entry of $A$ increases [13]. Therefore, since any matrix with nonzero codiagonal entries is an irreducible matrix, the largest eigenvalue of any symmetric nonnegative matrix with nonzero codiagonal entries is the spectral radius of the matrix and increases when any entry increases.

Corollary 1. The spectral radius of $S_{1}(\mathbf{d})$ is the largest eigenvalue of $Q\left(\mathcal{H}\left\{\mathcal{B}_{k}\right\}\right)$.

Proof. We have that the largest eigenvalues of $S_{i}(\mathbf{d})$ is $\rho\left(S_{i}(\mathbf{d})\right)$. From Theorem 1, the eigenvalues of $Q\left(\mathcal{H}\left\{\mathcal{B}_{k}\right\}\right)$ are the eigenvalues of the matrices $T_{j}, j \in \Omega$, together with the eigenvalues of the matrices $S_{i}(\mathbf{d})$. From the Cauchy's Interlace Theorem for eigenvalues of Hermitian matrices [6] and [8], the eigenvalues of each $T_{j}$ interlace the eigenvalues of each $S_{i}(\mathbf{d})$. Then the largest eigenvalue of $Q\left(\mathcal{H}\left\{\mathcal{B}_{k}\right\}\right)$ is $\max \left\{\rho\left(S_{i}(\mathbf{d})\right): i=1, \ldots, r\right\}$. Since 
$d_{k}+q_{1}(\mathcal{H}) \geq d_{k}+q_{i}(\mathcal{H})$ for $i=2, \ldots, r$, it follows $\rho\left(S_{1}(\mathbf{d})\right) \geq \rho\left(S_{i}(\mathbf{d})\right)$ for $i=2, \ldots, r$. Therefore $\max \left\{\rho\left(S_{i}(\mathbf{d})\right): i=1, \ldots, r\right\}=\rho\left(S_{1}(\mathbf{d})\right)$ and the proof is complete.

From now on, let $\mathcal{H}=\mathcal{C}_{r}$ with $r=2 s+1$. Since $q_{1}\left(\mathcal{C}_{r}\right)=4$, from Corollary 1 , we obtain

Corollary 2. The spectral radius of the $k \times k$ matrix

$$
\left[\begin{array}{ccccc}
1 & \sqrt{d_{2}-1} & & & \\
\sqrt{d_{2}-1} & d_{2} & \ddots & & \\
& \ddots & \ddots & \sqrt{d_{k-1}-1} & \\
& & \sqrt{d_{k-1}-1} & d_{k-1} & \sqrt{d_{k}} \\
& & & \sqrt{d_{k}} & d_{k}+4
\end{array}\right]=Q_{0}(\mathbf{d})
$$

is the largest signless Laplacian of $\mathcal{C}_{r}\left\{\mathcal{B}_{k}\right\}$.

\section{An upper bound on $q_{1}(\mathcal{G})$ in terms of $\Delta$ and $k(\mathcal{G})$}

From now on, let $k=k(\mathcal{G})$ and let $\mathcal{B}_{k}(\Delta)$ be the generalized Bethe tree with vertex degree sequence

$$
\mathbf{d}=(1, \Delta, \Delta, \ldots, \Delta, \Delta-2),
$$

from the pendant vertices to the root. Then each tree $\mathcal{T}_{i}$ is an induced subgraph of $\mathcal{B}_{k}(\Delta)$. Let $\mathcal{C}_{r}\left\{\mathcal{B}_{k}(\Delta)\right\}$ be the unicyclic graph obtained from $\mathcal{C}_{r}$ and $r$ copies of $\mathcal{B}_{k}(\Delta)$ by identifying the root of the $i-t h$ copy with the $i-t h$ vertex of $\mathcal{C}_{r}$. Hence $\mathcal{G}$ is an induced subgraph of $\mathcal{C}_{r}\left\{\mathcal{B}_{k}(\Delta)\right\}$ and consequently $q_{1}(\mathcal{G}) \leq q_{1}\left(\mathcal{C}_{r}\left\{\mathcal{B}_{k}(\Delta)\right\}\right)$.

Example 2. For instance the graph $\mathcal{G}$ in Example 1 is an induced subgraph of $\mathcal{C}_{3}\left\{\mathcal{B}_{4}(4)\right\}$ :

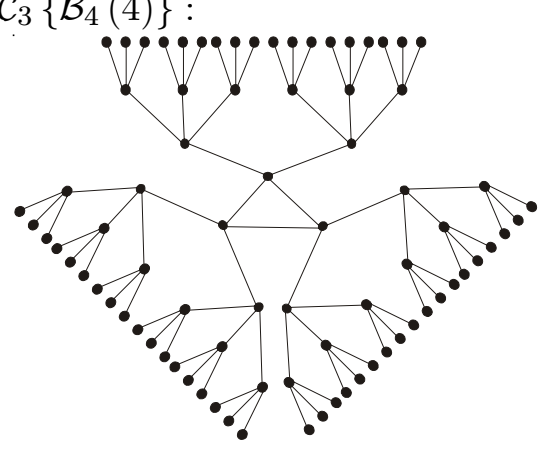


Applying Corollary 2 to the graph $\mathcal{C}_{r}\left\{\mathcal{B}_{k}(\Delta)\right\}$, we obtain that $q_{1}\left(\mathcal{C}_{r}\left\{\mathcal{B}_{k}(\Delta)\right\}\right)$ is equal to the spectral radius of the $k \times k$ matrix

(2.1) $Q_{0}(\Delta)=\left[\begin{array}{ccccc}1 & \sqrt{\Delta-1} & & & \\ \sqrt{\Delta-1} & \Delta & \ddots & & \\ & \ddots & \ddots & \sqrt{\Delta-1} & \\ & & \sqrt{\Delta-1} & \Delta & \sqrt{\Delta-2} \\ & & & \sqrt{\Delta-2} & \Delta+2\end{array}\right]$.

That is $q_{1}\left(\mathcal{C}_{r}\left\{\mathcal{B}_{k}(\Delta)\right\}\right)=\rho\left(Q_{0}(\Delta)\right)$.

Theorem 2. Let $\mathcal{G}$ be an odd unicyclic graph with $\Delta \geq 3$

$$
q_{1}(\mathcal{G})<\Delta+2 \sqrt{\Delta-1} \cos \frac{\pi}{2 k(\mathcal{G})+1} .
$$

Proof. We know that $q_{1}(\mathcal{G}) \leq q_{1}\left(\mathcal{C}_{r}\left\{\mathcal{B}_{k}(\Delta)\right\}\right)=\rho\left(Q_{0}(\Delta)\right)$. From $(2.1)$

$$
\mathrm{A}_{k}(\Delta)=\left[\begin{array}{ccccc}
0 & \sqrt{\Delta-1} & & \\
\sqrt{\Delta-1} & 0 & \ddots & & \\
& \ddots & \ddots & \sqrt{\Delta-1} & \\
& & \sqrt{\Delta-1} & 0 & \sqrt{\Delta-2} \\
& & & \sqrt{\Delta-2} & 2
\end{array}\right],
$$

with strict inequality in position $(1,1)$. It follows

$$
q_{1}(\mathcal{G}) \leq \rho\left(Q_{0}(\Delta)\right)<\Delta+\rho\left(A_{k}(\Delta)\right) .
$$

In order to prove $(2.2)$, we search for an upper bound on $\rho\left(A_{k}(\Delta)\right)$. Suppose $\Delta \geq 5$. Then

$$
\begin{aligned}
& \mathrm{A}_{k}(\Delta) \leq\left[\begin{array}{ccccc}
0 & \sqrt{\Delta-1} & & & \\
\sqrt{\Delta-1} & 0 & \sqrt{\Delta-1} & & \\
& \sqrt{\Delta-1} & \ddots & \ddots & \\
& & \ddots & 0 & \sqrt{\Delta-1} \\
& & & \sqrt{\Delta-1} & \sqrt{\Delta-1}
\end{array}\right] \\
& =D_{k}(\sqrt{\Delta-1})
\end{aligned}
$$


with strict inequalities in positions $(k-1, k)$ and $(k, k-1)$. Then

$$
\rho\left(A_{k}(\Delta)\right)<\rho\left(D_{k}(\sqrt{\Delta-1})\right) .
$$

The spectral radius of $D_{k}(\sqrt{\Delta-1})[9]$ is

$$
\rho\left(D_{k}(\sqrt{\Delta-1})\right)=2 \sqrt{\Delta-1} \cos \frac{\pi}{2 k+1} .
$$

Hence, for $\Delta \geq 5$,

$$
\rho\left(A_{k}(\Delta)\right)<2 \sqrt{\Delta-1} \cos \frac{\pi}{2 k+1} .
$$

Using this result in (2.3), we obtain

$$
q_{1}(\mathcal{G})<\Delta+2 \sqrt{\Delta-1} \cos \frac{\pi}{2 k+1}
$$

whenever $\Delta \geq 5$. We now study the cases $\Delta=3$ and $\Delta=4$. For $j=$ $1,2, . ., k$, let $a_{j}(\lambda)$ and $d_{j}(\lambda)$ be the characteristic polynomials of the $j \times j$ leading principal submatrices of $A_{k}(\Delta)$ and $D_{k}(\sqrt{\Delta-1})$, respectively. We have

$$
a_{k}(\lambda)=\operatorname{det}\left(\lambda I-A_{k}(\Delta)\right)
$$

and

$$
d_{k}(\lambda)=\operatorname{det}\left(\lambda I-D_{k}(\sqrt{\Delta-1})\right) .
$$

For $j=1,2, \ldots, k-1$, the $a_{j}(\lambda)$ and $d_{j}(\lambda)$ are identical polynomials.

Expanding along the last rows of $\operatorname{det}\left(\lambda I-A_{k}(\Delta)\right)$ and $\operatorname{det}\left(\lambda I-D_{k}(\sqrt{\Delta-1})\right)$, we obtain

$$
a_{k}(\lambda)=(\lambda-2) a_{k-1}(\lambda)-(\Delta-2) a_{k-2}(\lambda)
$$

and

$$
d_{k}(\lambda)=(\lambda-\sqrt{\Delta-1}) a_{k-1}(\lambda)-(\Delta-1) a_{k-2}(\lambda) .
$$

Sustracting (2.5) from (2.4), we get

$$
a_{k}(\lambda)-d_{k}(\lambda)=(\sqrt{\Delta-1}-2) a_{k-1}(\lambda)+a_{k-2}(\lambda)
$$


Since the eigenvalues of any tridiagonal symmetric tridiagonal matrix with nonzero codiagonal entries are simple eigenvalues, we may write

$$
a_{k}(\lambda)=\left(\lambda-\alpha_{1}\right)\left(\lambda-\alpha_{2}\right) \ldots . .\left(\lambda-\alpha_{k-1}\right)\left(\lambda-\alpha_{k}\right)
$$

where

$$
\alpha_{k}<\alpha_{k-1}<\ldots<\alpha_{2}<\alpha_{1}=\rho\left(A_{k}(\Delta)\right)
$$

are the zeros of the polynomial $a_{k}(\lambda)$. Let $\delta=\rho\left(D_{k}(\sqrt{\Delta-1})\right)$. We know that

$$
\delta=2 \sqrt{\Delta-1} \cos \frac{\pi}{2 k+1}
$$

is the largest zero of $d_{k}(\lambda)$. Let $\beta_{1}$ be the largest zero of the identical polynomials $d_{k-1}(\lambda)$ and $a_{k-1}(\lambda)$. Since the zeros of these polynomials strictly interlace the zeros of the polynomials $a_{k}(\lambda)$ and $d_{k}(\lambda)$, we obtain that $\alpha_{2}<\beta_{1}<\alpha_{1}$ and $\beta_{1}<\delta$. Therefore $\alpha_{2}<\delta, a_{k-1}(\delta)>0$ and

$$
\begin{aligned}
a_{k}(\delta) & =\left(\delta-\alpha_{1}\right)\left(\delta-\alpha_{2}\right) \ldots . .\left(\delta-\alpha_{k-1}\right)\left(\delta-\alpha_{k}\right) \\
& =\left(\delta-\alpha_{1}\right) P
\end{aligned}
$$

where $P>0$. Then $\rho\left(A_{k}(\Delta)\right)=\alpha_{1}<\delta$ if $a_{k}(\delta)>0$. From (2.5) and (2.6),

$$
(\delta-\sqrt{\Delta-1}) a_{k-1}(\delta)-(\Delta-1) a_{k-2}(\delta)=0
$$

and

$$
a_{k}(\delta)=(\sqrt{\Delta-1}-2) a_{k-1}(\delta)+a_{k-2}(\delta)
$$

Then

$$
a_{k}(\delta)=\left(\sqrt{\Delta-1}-2+\frac{\delta-\sqrt{\Delta-1}}{\Delta-1}\right) a_{k-1}(\delta) .
$$

Let $\Delta=4$. From (2.7)

$$
a_{k}(\delta)=\left(\sqrt{3}-2+\frac{2 \sqrt{3} \cos \frac{\pi}{2 k+1}-\sqrt{3}}{3}\right) a_{k-1}(\delta)
$$




$$
\begin{aligned}
& =\left(\frac{2 \sqrt{3}}{3}-2+\frac{2 \sqrt{3}}{3} \cos \frac{\pi}{2 k+1}\right) a_{k-1}(\delta) \\
& \geq\left(\frac{2 \sqrt{3}}{3}-2+\frac{2 \sqrt{3}}{3} \cos \frac{\pi}{5}\right) a_{k-1}(\delta) \\
& >0.08 a_{k-1}(\delta)>0 .
\end{aligned}
$$

Let now $\Delta=3$ and $k \geq 4$. From (2.7)

$$
\begin{aligned}
a_{k}(\delta) & =\left(\sqrt{2}-2+\frac{2 \sqrt{2} \cos \frac{\pi}{2 k+1}-\sqrt{2}}{2}\right) a_{k-1}(\delta) \\
& =\left(\frac{\sqrt{2}}{2}-2+\sqrt{2} \cos \frac{\pi}{2 k+1}\right) a_{k-1}(\delta) \\
& \geq\left(\frac{\sqrt{2}}{2}-2+\sqrt{2} \cos \frac{\pi}{9}\right) a_{k-1}(\delta) \\
& >0.03 a_{k-1}(\delta)>0 .
\end{aligned}
$$

It follows that (2.2) also holds when $\Delta=4$ and when $\Delta=3$ with $k \geq 4$. It remains to prove (2.2) when $\Delta=3$ with $k=2$ and $k=3$. We know that $q_{1}\left(\mathcal{C}_{r}\left\{\mathcal{B}_{2}(3)\right\}\right)$ is the largest eigenvalue of

$$
Q_{2}(3)=\left[\begin{array}{ll}
1 & 1 \\
1 & 5
\end{array}\right]
$$

and that $q_{1}\left(\mathcal{C}_{r}\left\{\mathcal{B}_{3}(3)\right\}\right)$ is the largest eigenvalue of

$$
Q_{3}(3)=\left[\begin{array}{ccc}
1 & \sqrt{2} & 0 \\
\sqrt{2} & 3 & 1 \\
0 & 1 & 5
\end{array}\right] .
$$

To four decimal places

$$
\rho\left(Q_{2}(3)\right)=5.2361<3+2 \sqrt{2} \cos
$$

and

$$
\rho\left(Q_{3}(3)\right)=5.4893<3+2 \sqrt{2} \cos
$$

The proof is complete.

The following corollary, in which the Hu's upper bound (1.1) is extended to an upper bound on $q_{1}(\mathcal{G})$, is an immediate consequence of Theorem 2. 
Corollary 3. Let $\mathcal{G}$ be an odd unicyclic graph with $\Delta \geq 3$. Then

$$
q_{1}(\mathcal{G})<\Delta+2 \sqrt{\Delta-1} .
$$

\section{References}

[1] D. Cvetkovic, P. Rowlinson, S. K. Simic, Signless Laplacian of finite graphs, Linear Algebra Appl. 423, pp. 155-171, (2007).

[2] D. Cvetkovic, P. Rowlinson, S. K Simic, Eigenvalue bounds for the signless Laplacian, Publications de L'institute Mathématique, Nouvelle série tome 81(95), pp. 11-27, (2007).

[3] R. Diestel, Graph Theory, Electronic Editions 2005, Springer-Verlag Hiedlberg, New York.

[4] G. H. Golub and C. F. van Loan, Matrix Computations, 2nd ed. ,John Hopkins University Press, (1989).

[5] C. S. Oliveira, L. S. de Lima, N. M. M. de Abreu, P. Hansen, Bounds on the index of the signless Laplacian of a graph, Discrete Applied Mathematics 158, pp. 355-360, (2010).

[6] R. A. Horn, C. R. Johnson, Matrix Analysis, Cambridge University Press, Cambridge, (1991).

[7] S. Hu, The largest eigenvalue of unicyclic graphs, Discrete Math. 307, pp. 280-284, (2007).

[8] Y. Ikebe, T. Inagaki, S. Miyamoto, The Monotonicity Theorem, Cauchy's Interlace Theorem and Courant-Fisher Theorem, American Mathematical Monthly Vol. 94, No. 4, April, pp. 352-354, (1987).

[9] S. Kouachi, Eigenvalues and eigenvectors of tridiagonal matrices, Electronic Journal of Linear Algebra 15, pp. 115-133, (2006).

[10] O. Rojo, New upper bounds on the spectral radius of unicyclic graphs, Linear Algebra Appl. 428, pp. 754-764, (2008).

[11] O. Rojo, Spectra of copies of a generalized Bethe tree atached to any graph, Linear Algebra Appl. 431, pp. 863-882, (2009). 
[12] L. N. Trefethen and D. Bau, III Numerical Linear Algebra, Society for Industrial and Applied Mathematics, (1997).

[13] R. Varga, Matrix Iterative Analysis, Theory, Prentice-Hall, Inc., (1965).

\section{Macarena Collao}

Departamento de Matemáticas

Universidad Católica del Norte

Casilla 1280

Antofagasta

Chile

e-mail : maca_collaom@hotmail.com

\section{Pamela Pizarro}

Departamento de Matemáticas

Universidad Católica del Norte

Casilla 1280

Antofagasta

Chile

e-mail : parkinzon_triste@hotmail.com

and

\section{Oscar Rojo}

Departamento de Matemáticas

Universidad Católica del Norte

Casilla 1280

Antofagasta

Chile

e-mail : orojo@ucn.cl 